\title{
Detailed Analysis of $\alpha, \omega$-Bis(4-hydroxybutyl) Poly(dimethylsiloxane) Using GPC-MALDI TOF Mass Spectrometry
}

\author{
E. Peter Maziarz, III, X. Michael Liu, Edmond T. Quinn, Yu-Chin Lai, \\ Daniel M. Ammon, Jr., and George L. Grobe, III \\ Bausch and Lomb Inc., Rochester, New York, USA
}

\begin{abstract}
In this study the prepolymer $\alpha$, $\omega$-bis(4-hydroxybutyl) poly(dimethylsiloxane), used in the formulation of oxygen permeable films, is evaluated by gel permeation chromatography (GPC) combined with matrix assisted laser desorption ionization (MALDI) time of flight (TOF) mass spectrometry (MS). Two unexpected mass distributions are observed in the mass spectra. Reaction schemes for the formation of these distributions are proposed. A solution phase trimethylsilane end group modification was performed on the prepolymer to determine whether the unexpected mass distributions occur as impurities from synthesis or as artifacts from the MS process. Evaluation of the TMS modified prepolymer indicates the unexpected mass distributions indeed occur as impurities from the synthetic procedure. Average molecular weight values are determined by traditional GPC, direct MALDI-TOF MS, and GPCMALDI-TOF MS methods and the results are compared. (J Am Soc Mass Spectrom 2002, 13, 170-176) @ 2002 American Society for Mass Spectrometry
\end{abstract}

0 iloxane polymers have unique properties such as low surface energy, low glass transition temperature, good thermal stability, and high gas permeability [1]. Synthetic materials containing polysiloxane prepolymer segments play pivotal roles in many personal care [2,3] and biomedical products [4]. The ability to manufacture such products having physico-chemical and mechanical properties within desired specifications often depend on the batch-to-batch consistency of the prepolymers (here prepolymer refers to a polymeric reagent that can undergo further reaction to form longer chain polymers) used in the product formulation. Gel permeation chromatography (GPC) can be used to determine average molecular weight values for polymer products. Automated GPC systems can provide convenient and efficient analysis of several batches of a particular polymer product, thus enabling quality control protocols to be adopted. However GPC does not provide information regarding prepolymer repeat unit sequence or end group chemistry. Testimony to the importance of such detail is noticed when a particular prepolymer falls within MWD specifications, but the final product fails mechanical testing. The incidence of this scenario increases with products that require more narrow limits of specification. Thus it is important to utilize additional analytical techniques that provide

Published online December 3, 2001

Address reprint requests to Dr. E. P. Maziarz, III, Bausch \& Lomb Inc., 1400 North Goodman Street, Rochester, NY 14603-0450, USA. E-mail: e_peter_maziarz@bausch.com detailed information of polymeric materials. Recently, detailed information including repeat unit sequence and end group chemistry of polymeric materials has been obtained from electrospray ionization (ESI) [5-11] and matrix assisted desorption/ionization (MALDI) [12-19] mass spectrometry (MS) techniques. Several reviews [20-24] and comparisons [25-29] of these MS techniques for polymer analysis appear in the literature. In this report MALDI time of flight (TOF) MS is used to evaluate the prepolymer $\alpha, \omega$-bis(4-hydroxybutyl) poly(dimethylsiloxane) HB-PDMS (1), that is used to make oxygen permeable films.

In general there are some caveats to prepolymer analysis by MALDI-TOF MS. First, direct MALDI-TOF MS analysis of polydisperse (PD $>1.1$ ) polymers suffers from mass bias effects such that the high-mass oligomers in the MWD are under represented in the mass spectrum [30-34]. In some instances, the relatively high mass oligomers that are predicted by GPC data are entirely attenuated in the mass spectrum. This mass bias effect limits the amount of qualitative data that can be obtained from analysis, especially when comparing impurity levels from different average mass prepolymer blends [34]. Second, there may be some uncertainty as to whether unexpected mass distributions observed in the mass spectrum occur as impurities from synthesis or as artifacts from the sample preparation or MS process. Such artifacts could include reaction of the prepolymer with the matrix $[20,35,36]$ or complexation to unexpected charge agents, [37, 38] fragmentation from high or low mass oligomer molecules, [35] or from 
inter- and intra-molecular gas-phase interactions (i.e., backbiting). This is especially a concern for analysis of prepolymers since they usually contain relatively reactive end groups. To properly determine material specifications it is of paramount importance to identify and be certain of the origin (impurity from synthetic procedure or instrumental artifact) of unexpected mass distributions observed from analysis of the prepolymer.

In this study the combination of GPC and MALDITOF MS is used to evaluate an HB-PDMS prepolymer used in the production of oxygen permeable films.

\section{Experimental}

\section{Prepolymer Materials}

HB-PDMS prepolymer was synthesized in-house from a previously described procedure [3]. Trimethysilane end group modification of HB-PDMS was performed from a method previously described by Kanjolia et al. [39].

\section{Gel Permeation Chromatography}

The solvent delivery system utilized a Waters Alliance 2690 Separation Module (Waters Corporation, Milford, MA). A Waters 2410 refractive index detector was used to monitor the GPC effluent. Two mixed bed linear PL-gel columns (Polymer Laboratories, Amherst, MA) were used to perform the separation. The packing material had a particle size diameter of $3 \mu \mathrm{m}$. One hundred microliters of a $0.15 \%$ (wt/vol) sample solution was injected into the GPC system. HPLC grade tetrahydrofuran (Aldrich, St. Louis, MO) was used as the mobile phase and the flow rate was set at 1.0 $\mathrm{ml} / \mathrm{min}$. A series of narrow disperse polystyrene standards (Polymer Laboratories) were used to calibrate the GPC system. The calibration curve was constructed by plotting $\log M W$ of the calibration standards as a function of retention volumes using the third order polynomial fit. Millennium software with a GPC option (Waters Corporation) was used to control the GPC system and perform all the data manipulations. Fractions of GPC eluent were manually collected in separate scintillation vials approximately every thirty s. Aliquots from these vials were prepared for MALDI-TOF MS analysis.

\section{Mass Spectrometry}

The MALDI-TOF MS data was obtained with an Applied Biosystems Voyager DE-STR TOF (Framingham, MA) mass spectrometer, operating in the reflector mode. Ions were formed by laser desorption at $337 \mathrm{~nm}$ $\left(\mathrm{N}_{2}\right.$ laser, $3 \mathrm{~ns}$ pulse width, $10^{6} \mathrm{~W} / \mathrm{cm}^{2}, 100 \mu \mathrm{m}$ diameter spot), accelerated to $25 \mathrm{kV}$ and detected as positive ions. During the ionization process a delay time of 150 ns was applied before acceleration. Additionally, the grid and guide wire voltages were set at $70.0 \%$ and $0.010 \%$ of the applied acceleration voltage respectively to focus the beam of ions. Typically, 256 laser shots were averaged for each spectrum. Dithranol (Aldrich) was used as the sample matrix and was prepared as 20 $\mathrm{mg} / \mathrm{ml}$ in HPLC grade tetrahydrofuran (Aldrich) with the addition of $1 \%$ by volume $\mathrm{NaCl}$ (Aldrich). Matrix solution and HB-PDMS samples were mixed 10:1 and 1 $\mu \mathrm{L}$ of this mixture was manually spotted on the sample plate.

\section{Data Analysis}

Data analysis including average molecular weight values from GPC fractions was performed using GRAMS/ 386 (Galactic Industries, Salem, NH) software. The mass of an oligomer was determined from the monoisotopic peak in the isotope cluster distribution. For accurate mass measurements nominal 1250 Da methyl terminated PDMS (ME-PDMS) (Aldrich) was used as an internal standard. This standard was ideal because the intact ME-PDMS oligomers did not overlap with the sample oligomers. A two-point calibration was performed using ME-PDMS oligomers that bracketed the sample oligomers. Mass errors were determined from at least three measurements. The internal calibration spectra were 19 point smoothed before mass calibration. The accurate mass determinations were not used to unambiguously determine the elemental composition of the unknown distributions. Instead these calculations were used to help verify products proposed to occur from decomposition of HB-PDMS. The degree of polymerization $(n)$ was obtained by subtracting the monoisotopic masses of the end groups and sodium charge agent from the oligomer and dividing by the monoisotopic mass of the siloxane repeat unit (74.0182 Da).

\section{Spectroscopy}

Fourier transform infrared spectroscopy (FT-IR) data was collected on a Bio-RAD FTS155 spectrometer. The spectrometer was purged with dry $\mathrm{N}_{2}$ gas to eliminate interference of $\mathrm{CO}_{2}$ and water moisture. The instrument was calibrated with a standard polystyrene sample. Neat samples of HB-PDMS and TMS-PDMS were prepared between salt plates. Each FT-IR spectrum was the result of 64 scans at a nominal resolution of $4 \mathrm{~cm}^{-1}$.

\section{Results and Discussion}

Figure 1a and $b$ illustrate the GPC trace and MALDITOF mass spectrum of HB-PDMS that is used as a prepolymer in the production of oxygen permeable films. From the GPC trace average mass values of 1741 $\mathrm{Da}$ and $2206 \mathrm{Da}$ were calculated for $\mathrm{M}_{n}$ and $\mathrm{M}_{w}$ respectively. From these values a PD of 1.27 was determined. Two distinct distributions of peaks denoted as (filled diamond) and (filled square) are observed in Figure 1b. A third distribution, denoted as (filled circle), is observed at very low S/N. Oligomer peaks within each distribution differ by a mass nomi- 

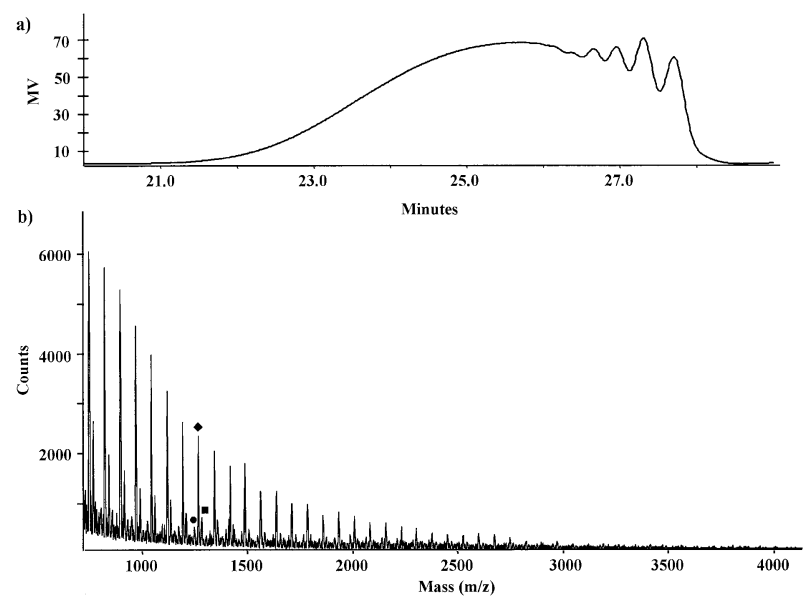

Figure 1. (a) GPC trace of HB-PDMS $\left(\mathrm{M}_{n}=1741, \mathrm{M}_{w}=2206, \mathrm{M}_{z}\right.$ $=2634, \mathrm{PD}=1.27)$. (b) Direct MALDI-TOF MS analysis of HB-PDMS. Three sodiated poly(dimethylsiloxy) mass distributions are denoted as (filled circle), (filled diamond), (filled square).

nally equivalent to a dimethylsiloxy $\left(\mathrm{C}_{2} \mathrm{H}_{6} \mathrm{SiO} ; 74 \mathrm{Da}\right)$ unit. Thus repeat unit homogeniety is observed across each distribution of peaks. The charging agent for each of the peaks in this mass spectrum was determined to be sodium cations. This was determined from experiments where lithium or potassium was used as charging agents and the three distributions shifted mass according to their differences with the sodium adducts. With the same charging agent for all three distributions, one could assume the mass difference between each distribution occurs exclusively from differences in end group composition. This observation raises some concern because the theoretical formulation of this particular oxygen permeable film requires the poly(dimethyl siloxane) prepolymer to have specific end group chemistries (butyl hydroxy) that facilitate reaction with additional segments.

The mass of the peaks in the most intense distribution, denoted by (filled diamond), is consistent with intact sodiated HB-PDMS (1) oligomers. Thus the peaks in the (filled diamond) distribution correspond to mass values of $\left[74 n+204+\mathrm{Na}^{+}\right]$, where $n$ is equal to the repeat unit integer and 204 is equal to the sum of the end group mass. This assignment was corroborated by a low average error of $5.2 \mathrm{ppm}( \pm 2.0 \mathrm{ppm})$ between monoisotopic masses of several oligomers within the (filled diamond) distribution and theoretical monoisotopic masses of compositions consistent with HB-PDMS oligomers. These results were obtained from internal standard calibration with ME-PDMS (data not illustrated). The distributions denoted by (filled square) and (filled circle) were not expected to occur and thus additional analytical efforts are required for their elucidation and to determine their extent of existence. However, the mass bias effects associated with direct analysis of PD polymers limit the amount of qualitative information that can be obtained from the MWD. For example the profile of peaks in Figure 1 indicate that the unexpected distributions (filled square) and (filled circle), hardly discernable, may occur predominantly within the low-mass region. Also by comparing relative peak intensities it appears that the (filled square) distribution occurs to a much greater extent than the (filled circle) distribution. This data may lead one to assume that a more pure HB-PDMS blend could be obtained from a simple but costly preparatory GPC method that could exclude the low-mass products from the stock prepolymer sample. For us this assumption would not be unreasonable since we have observed the formation of exclusively low to moderate mass side products from certain PDMS synthetic procedures. We also have observed for some PDMS polymers that the intensities of impurity peaks, with respect to one another, change as a function of mass throughout the MWD. This has been observed from PDMS products that contain branched oligomers within the moderate to high-mass region of the MWD [40, 41]. Direct analysis does not allow such detail to be examined with certainty since the intact higher mass products, predicted by the GPC data, are biased or not observed at all. This is especially problematic when comparing impurity levels from different average mass prepolymer blends. Furthermore we are fundamentally uncertain about the true origin of these unexpected distributions; are they true impurities from the synthetic procedure of HB-PDMS or are they generated as an artifact from the MS process (fragmentation of high or low mass HB-PDMS oligomers, gas-phase interactions, reaction with matrix or charge agent)? Since the information obtained from the MS data can have a profound impact on the prepolymers' ultimate function, it behooves us to initiate further studies that may eliminate these uncertainties.

\section{GPC-MALDI TOF MS Analysis}

One way to minimize the potential for uncertainties in polydisperse prepolymer analysis is to size-separate the $n$-mers within the MWD by passing them through a GPC column, thereby achieving nearly mono-disperse $(\mathrm{D}<1.1)$ fractions, prior to MS analysis [41-48]. Figure 2 illustrates the MALDI-TOF mass spectra of several fractions that contain GPC elution volumes from approximately 23.0 to $26.0 \mathrm{~min}$ for HB-PDMS (Mn 1500). The well-defined Gaussian distribution of peaks in each mass spectrum indicates a relative absence of mass bias effects observed in Figure 1 from direct analysis. This allows us to gain insight into the higher mass peaks in the MWD. From the data in Figure 2 we can observe that the (filled square) and (filled circle) mass distributions occur throughout the entire MWD and not exclusively at the low mass end. Thus if the (filled square) and (filled circle) distributions are truly impurities from synthesis then a more highly purified HB-PDMS sample will most likely require synthetic modification rather than simple preparatory column purification methods. We also observe that the relative intensities of the (filled square) peaks decrease with increasing mass 


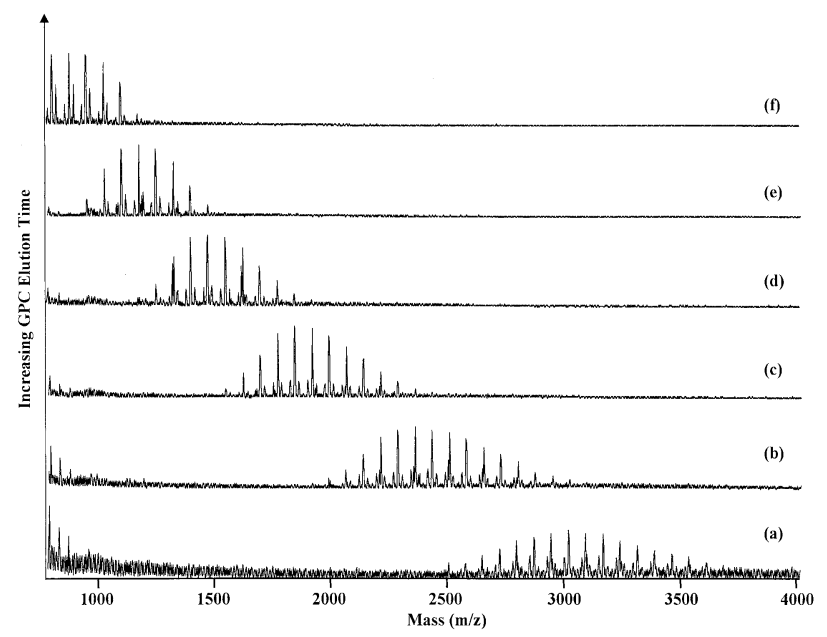

Figure 2. Analysis of HB-PDMS using GPC combined with MALDI-TOF MS. Post column fractions were manually collected prior to MS analysis. GPC fraction times are: (a) 23.0-23.5, b) 23.5-24.0, (c) 24.0-24.5, (d) 24.5-25.0, (e) 25.5-26.0, (f) 26.0-26.5 min. Each spectrum contains oligomers from approximately 0.5 $\mathrm{mL}$ of GPC elluent.

while the (filled circle) peaks increase with increasing mass. This provides us with a notion that (filled square) and (filled circle) products might be interactive. Additionally size separating precludes the possibility of a particular peak [from the (filled square) or (filled circle) distribution] occurring from fragmentation of a high or low-mass product outside the fractionated mass window. Thus we limit the existence of the two unexpected mass distributions (filled square) and (filled circle) to occur from one of three sources: (1) Reaction of the parent distribution with the matrix or charging reagent; (2) from inter- or intra-molecular gas-phase reactivity during the desorption/ionization process; or (3) as true impurities from the synthetic procedure.

\section{Elucidation of the (Filled Square) and (Filled Circle) Distributions}

Figure 3 illustrates the MALDI-TOF mass spectrum of a GPC fraction containing the elution volume from $\sim 24.0$ to $24.5 \mathrm{~min}$. The Figure 3 inset illustrates the resolved isotope peaks of representative $n$-mers from each distribution observed from analysis. The distribution denoted by (filled square) is proposed to be PDMS oligomers end capped with one butyl-hydroxy $\left(\mathrm{C}_{4} \mathrm{H}_{9} \mathrm{O}\right)$ and one hydroxy $(\mathrm{OH})$ end group, thus having a mass distribution equivalent to $\left[74 n+74+\mathrm{Na}^{+}\right]$. The formation of this product is proposed to occur from a combination of events. Mechanisms for these formations are illustrated in Scheme 1. First, polymer backbiting could occur where the reactive butyl hydroxy end group undergoes an intramolecular reaction with its nearest siloxane group ( $\mathrm{Si}-\mathrm{O})$ along the backbone of the polymer. This event results in the formation of the stable 1,1-dimethyl-1-sila-oxacyclohexane (DSO) compound and $\alpha$-hydroxy- $\omega$-butylhydroxy poly(dimethyl-

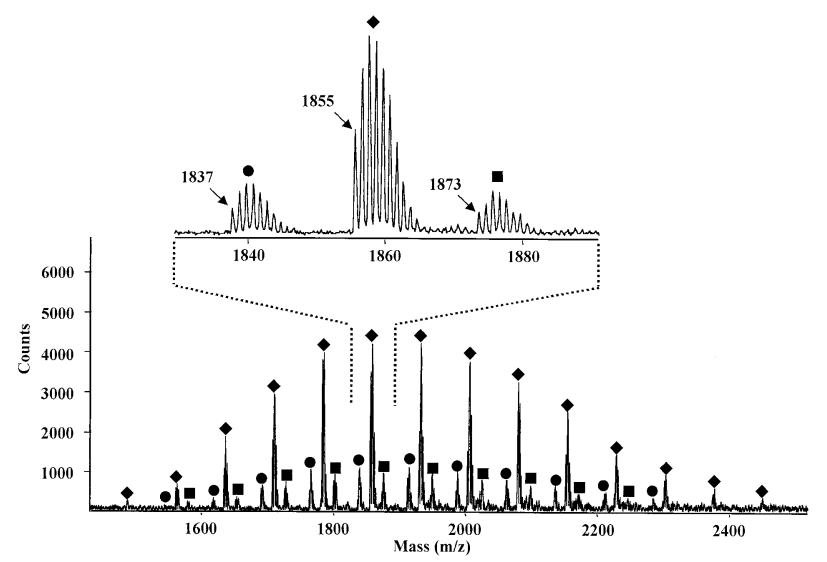

Figure 3. MALDI-TOF MS analysis of a GPC fraction containing the elution volume from 24.0 to $24.5 \mathrm{~min}$. The inset illustrates isotopically resolved (7000 RP) representative oligomer peaks from the three observed distributions [(filled circle) HBB-PDMS (3), $n=21 ; 1837$ Da nominal monoisotopic mass], [(filled diamond) HB-PDMS (1), $n=22 ; 1855$ Da nominal monoisotopic mass], and [(filled square) HBH-PDMS (2), $n=24 ; 1873 \mathrm{Da}$ nominal monoisotopic mass].

siloxane) HBH-PDMS (2). The second event involves reaction of a proton with the HB-PDMS polymer backbone to form two $n$-mers of HBH-PDMS. The isotopically resolved peaks (resolving power of 7000) in Figure 3 provide the ability to perform accurate mass measurements that help corroborate proposed compositions. After calibration with an internal standard, the compared experimental and theoretical mono-isotopic masses of the proposed composition HBH-PDMS (2) yielded a deviation of $6.8 \mathrm{ppm}( \pm 0.96 \mathrm{ppm})$. This error is within the instrumental specifications for internal standard calibration (30 $\mathrm{ppm}$ ) and thus provides strong evidence in support of $\left(\mathrm{Si}_{24} \mathrm{C}_{52} \mathrm{H}_{154} \mathrm{O}_{25} \mathrm{Na}\right)$ as the oli-

a)
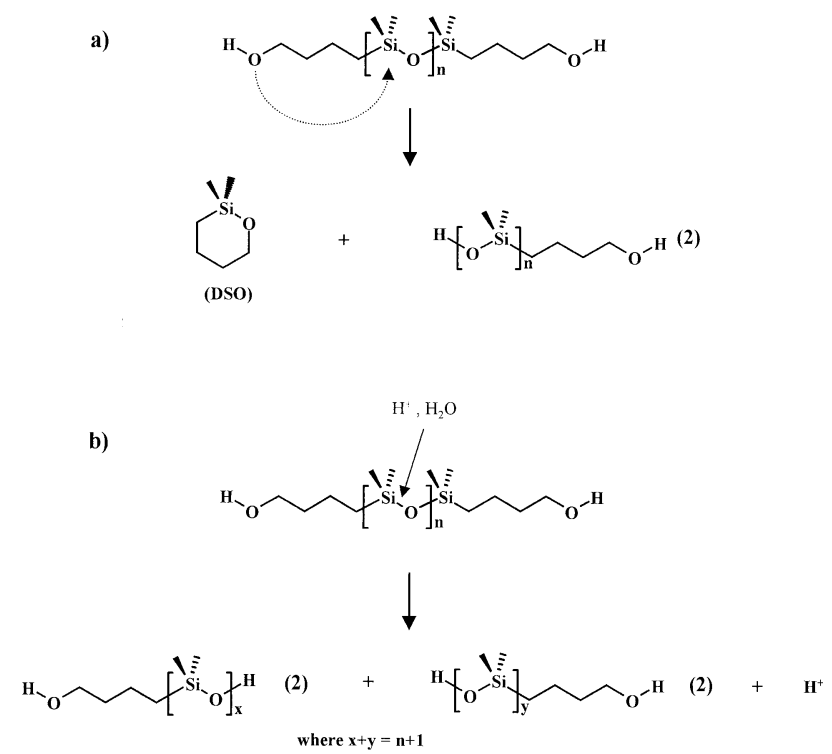

Scheme 1. Proposed (a) intramolecular backbiting reaction and (b) acid hydrolysis reaction to form $\alpha$-hydroxy, $\omega$-butylhydroxy poly(dimethylsiloxane) (HBH-PDMS) oligomers. 


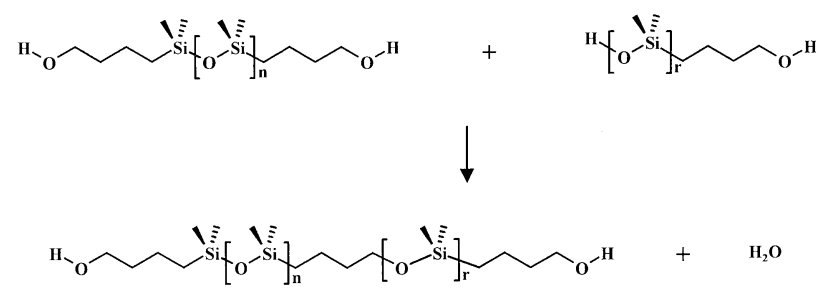

Scheme 2. Proposed condensation reaction of HB-PDMS with $\mathrm{HBH}-\mathrm{PDMS}$ to form $\alpha, \omega$-Bis(4-hydroxybutyl-Poly(dimethylsiloxane)butane (HBB-PDMS) oligomers.

gomer's proper identity. However it should be noted that this mass agreement is not sufficient to unambiguously prove the proposed elemental composition. Since each oligomer within the (filled square) distribution contains only one of the desired butyl hydroxy end groups, these oligomers are considered mono-functional. If in fact the (filled square) distribution occurs from the synthetic procedure, the degree of monofunctionality from batch-to-batch could have a significant impact on end product quality. Alternatively the distribution denoted by (filled square) could represent macro cyclic PDMS oligomers $\left(\mathrm{SiC}_{2} \mathrm{H}_{6} \mathrm{O}\right)_{n}$ having a mass distribution equivalent to $\left[74 n+\mathrm{Na}^{+}\right]$. It should be noted that a resolving power of approximately 35,000 would be required to resolve an HBH-PDMS and a macro cyclic PDMS oligomer.

The identity of the (filled circle) oligomers is proposed to be products having two butyl hydroxy end groups and one butyl group between PDMS n-mers thus having a mass distribution equivalent to [74n+ $260+\mathrm{Na}^{+}$]. A proposed mechanism for the formation of the (filled circle) oligomers is illustrated in Scheme 2. Formation of these $\alpha, \omega$-Bis(4-hydroxybutyl-Poly(dimethylsiloxane)butane HBB-PDMS (3) products could result from a condensation reaction where a hydroxy end group from a (filled square) oligomer interacts with a butylhydroxy end group of a (filled diamond) oligomer. Such condensation reactions are typical for silanol $(\mathrm{SiOH})$ containing compounds. $[49,50]$ From triplicate measurements, the compared theoretical and experimental monoisotopic masses resulted in a deviation of $13 \mathrm{ppm}( \pm 2.4 \mathrm{ppm})$. Thus the identity of the (filled circle) oligomers consistent with the HBB-PDMS composition proposed in Scheme 2 must be considered.

\section{Solution-Phase End Group Modification of HB-PDMS}

One way to determine whether the (filled square) and (filled circle) distributions are generated as impurities from the synthetic procedure or as instrumental artifacts is to perform a solution phase end group modification that converts the reactive hydroxy end groups to less reactive trimethyl silane (TMS) end groups. The unreactive TMS groups from the end group modified PDMS prepolymer would not be expected to undergo a gas-phase backbiting mechanism analogous to the

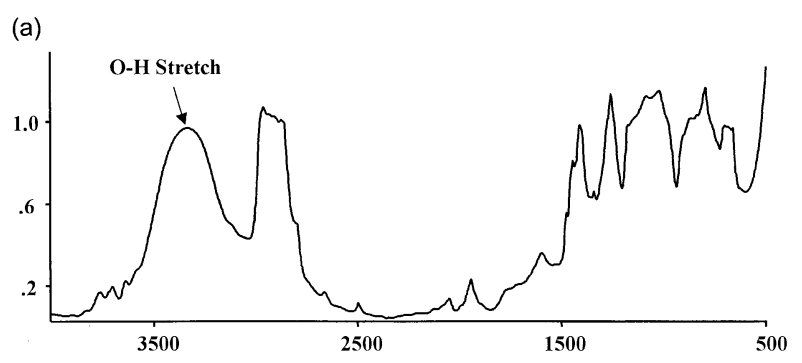

(b)

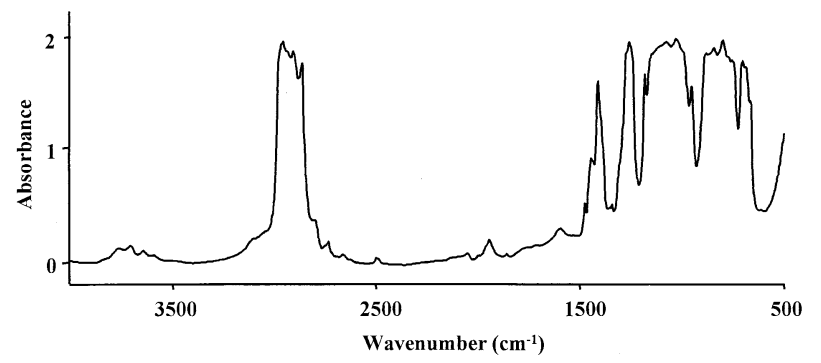

Figure 4. FT-IR spectra of (a) HB-PDMS; observation of the $\mathrm{OH}$ stretching frequency $\left(\sim 3300 \mathrm{~cm}^{-1}\right)$ and (b) TMS-PDMS; no $\mathrm{OH}$ stretching frequency observed.

mechanism illustrated in Scheme 1 for HB-PDMS. Additionally a gas phase condensation reaction, as proposed in Scheme 2, could not occur with the TMSPDMS sample. Thus if the (filled square) and (filled circle) distributions, Figure 3, occur as instrumental artifacts from gas phase interactions, then the TMSPDMS mass spectrum should have a single distribution of $\alpha, \omega$-bis-trimethylsiloxybutyl PDMS, TMS-PDMS, (4).

However if the (filled square) and (filled circle) distributions occur as impurities from the HB-PDMS synthetic procedure, one would expect the TMS-PDMS mass spectrum to have three distributions, consistent with Figure 3, however the mass shifted according to the TMS end group modification. For example a $-4 \mathrm{Da}$ mass shift is expected for replacement of two $\mathrm{OH}$ groups on the $n-2$ oligomers. A solution phase reaction of the HB-PDMS prepolymer with hexamethyldisilazane using a method previously described by Kanjolia et al. was performed [39]. This reaction converts the hydroxy end groups of HB-PDMS to TMS end groups. Figure 4 illustrates FT-IR spectra of the HB-PDMS prepolymer before and after TMS modification. The intense broad band at $3300 \mathrm{~cm}^{-1}$ in Figure 4a indicates the $\mathrm{OH}$ stretch from the hydroxy end groups of HBPDMS. The absence of this $\mathrm{OH}$ stretch peak in Figure $4 \mathrm{~b}$ indicates nearly complete conversion of $\mathrm{OH}$ to TMS end groups. Figure $5 \mathrm{a}$ and $\mathrm{b}$ illustrate insets from the MALDI-TOF mass spectra of HB-PDMS and TMSPDMS respectively. Three different mass distributions, denoted as (open circle), (open diamond), and (open square) are indeed observed in the MALDI-TOF mass spectrum of TMS-PDMS. Comparison of the representative oligomers in Figure $5 \mathrm{a}$ indicates that each oligomer in Figure $5 \mathrm{~b}$ is mass shifted by $-4 \mathrm{Da}$ according to TMS end group modification of $n-2$ oligomers. This 

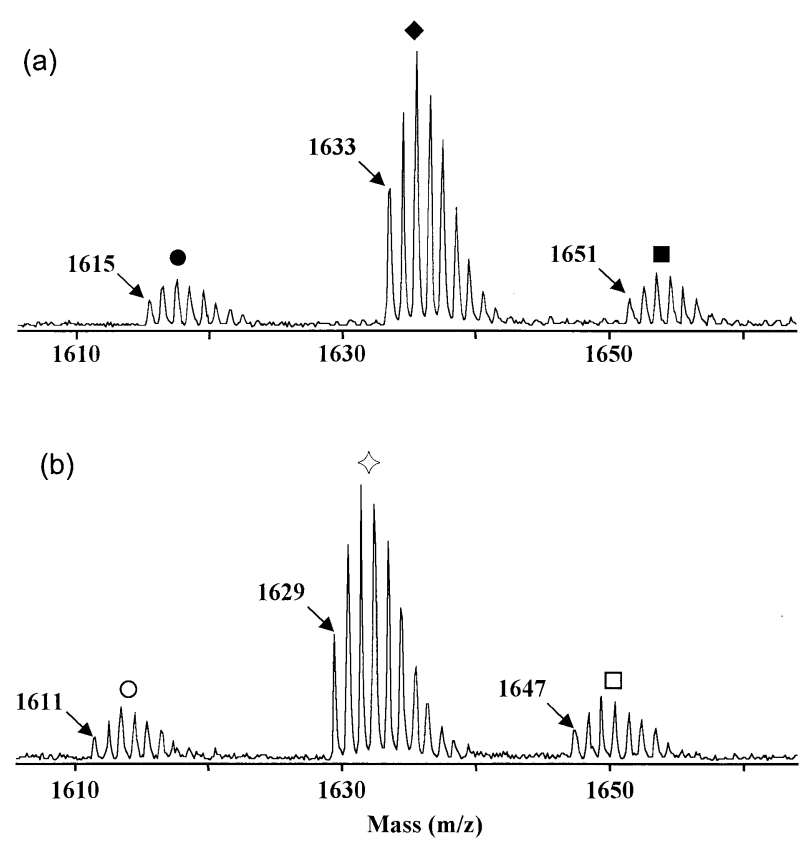

Figure 5. MALDI-TOF MS spectra of similar GPC fractions from (a) HB-PDMS; [(filled circle)-HBB-PDMS (3), (filled diamond)-HBPDMS (1), (filled square)-HBH-PDMS (2)] and (b) TMS-PDMS; distributions denoted as (open circle), (open diamond), and (open square) represent TMS end group modified HBB-PDMS, HBPDMS (4), and HBH-PDMS respectively.

indicates the (filled square) and (filled circle) distributions must have existed as part of the solutionphase prepolymer sample prior to TMS modification. Additionally, since the same sample preparation conditions were used for HB-PDMS and TMS-PDMS sample, it is clear that the (filled square) and (filled circle) distributions do not occur from reaction of matrix, solvent, or charge reagent. From this data it can be concluded the (filled square) and (filled circle) distributions occur as solution phase products and thus are true impurities from the HB-PDMS synthetic procedure.

\section{Determination of Average Mass Values for HB-PDMS}

Average mass values for HB-PDMS were obtained from traditional GPC calibrated with polystyrene standards, direct MALDI-TOF MS (Figure 1), from a summation of mass spectra of HB-PDMS fractions, and from GPC calibrated with mass values from HB-PDMS fractions (data from Figure 2). The determined average mass values and percent difference comparisons are summarized in Table 1. Relative to the other techniques used in this study, direct MALDI-TOF MS underestimates the PD by approximately $10 \%$. The average molecular weights from direct MALDI-TOF MS are lower than that obtained by traditional GPC and the differences between $\mathrm{M}_{n}, \mathrm{M}_{w}$, and $\mathrm{M}_{z}$ become increasingly larger. This can be explained by the fact that higher order average mass values such as $\mathrm{M}_{w}$ and $\mathrm{M}_{z}$ are significantly influenced by the higher mass oligomers in the MWD. This data indicates that direct MALDI-TOF MS analysis underestimates the higher molecular weight oligomers even for relatively narrow disperse polymers. Such discrimination results in an underestimation of the MWD for the polymer sample [51].

The PD values determined from the two GPCMALDI TOF MS techniques are within $1 \%$ of that determined by traditional GPC. The average molecular weights obtained from both methods of GPC-MALDITOF MS techniques agree reasonably well with each other. Although the average molecular weights are lower than that obtained by traditional GPC, the differences are consistent from $\mathrm{M}_{n}$ to $\mathrm{M}_{z}$. This indicates that the high and low mass products in the MWD are represented equally by GPC-MALDI TOF MS methods. Also the lower molecular weights determined by GPC-MALDI TOF MS suggests that PDMS has a different hydrodynamic volume than PS in the THF system.

Table 1. Comparison of average molecular weight values for HB-PDMS

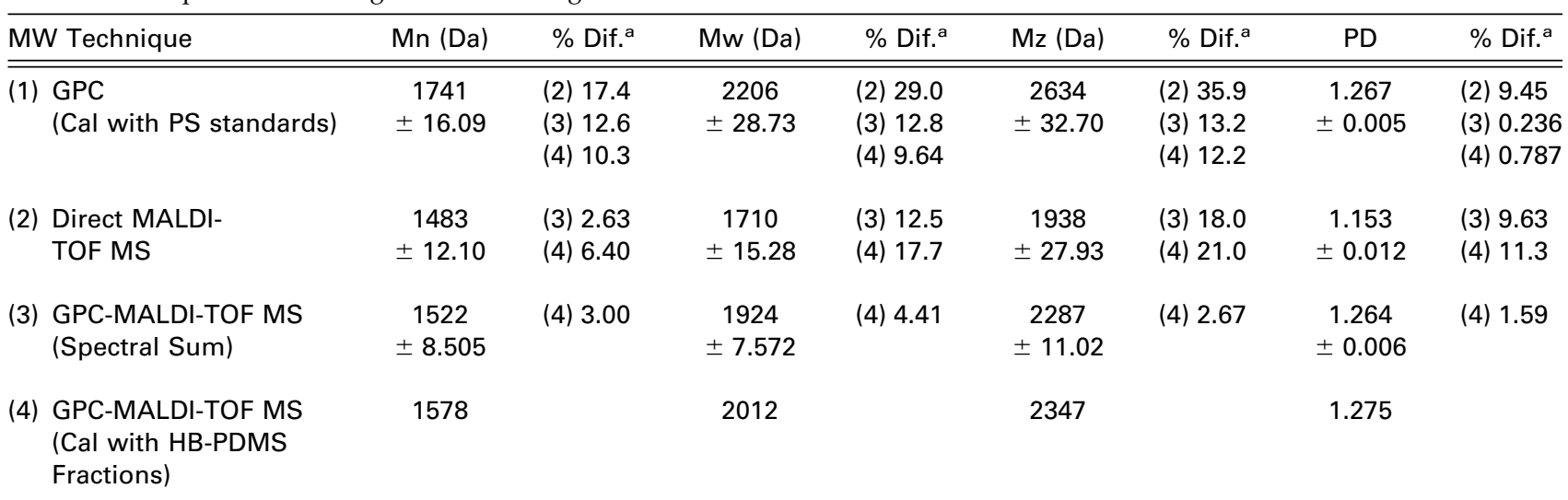

a Under the "\% Dif." (or \% Difference) columns, numbers in parentheses indicate the compared MW method. 


\section{Conclusions}

In this report a polydisperse prepolymer $\alpha, \omega$-bis(4hydroxybutyl) poly(dimethylsiloxane) HB-PDMS (1), used in the formulation of oxygen permeable films, was evaluated by direct MALDI-TOF MS and by the combination of GPC with MALDI-TOF MS analysis. Collectively, this information provided us a more detailed understanding of the underlying principles of HBPDMS synthesis.

\section{Acknowledgments}

The authors thank Gary Baker, Troy Wood, and Dave Heiler for helpful suggestions. Acknowledgment is made to Bausch \& Lomb for support of this research.

\section{References}

1. Carraher, C., Jr. Polymer Chemistry. Marcel Dekker: New York, 1996, pp 363-365.

2. Kunzler, J. F. TRIP 1996, 4, 52-59.

3. Lai, Y. C.; Ozark, R.; Quinn, E. T. J. Polym. Sci. Chem. Part A 1995, 33, 1773-1782.

4. Nelson, G. D. Plast. Reconstr. Surg. 1980, 66, 969-970.

5. Yalcin, T.; Gabryelski, W.; Li, L. Anal. Chem. 2000, 72, 38473852.

6. Maziarz, E. P., III; Baker, G. A.; Wood, T. D. Macromolecules 1999, 32, 4411-4418.

7. Koster, S.; Duursma, M. C.; Boom, J. J.; Heeren, R. M. J. Am. Soc. Mass Spectrom. 2000, 11, 536-543.

8. O'Connor, P. B.; McLafferty, F. W. J. Am. Chem. Soc. 1995, 117, 12826-12831.

9. Shi, S. D.-H.; Hendrickson, C. L.; Marshall, A. G.; Simonsick, W. J., Jr.; Aaserud, D. J. Anal. Chem. 1998, 70, 3220-3226.

10. Mahon, A.; Kemp, T. J.; Buzy, A.; Jennings, K. R. Polymer 1997, $38,2337-2349$.

11. Hunt, S. M.; Binns, M. R.; Sheil, M. M. J. Appl. Polym. Sci. 1995, 56, 1589-1597.

12. Pasch, H.; Gores, F. Polymer 1995, 10, 1999-2005.

13. Jackson, A. T.; Jennings, K. R.; Scrivens, J. H. J. Am. Soc. Mass Spectrom. 1997, 8, 76-85.

14. Danis, P. O.; Karr, D. E.; Simonsick, W. J., Jr.; Wu, D. T. Macromolecules 1995, 28, 1229-1232.

15. Montaudo, G.; Montaudo, M. S.; Puglisi, C.; Samperi, F. Macromolecules 1995, 28, 4562-4569.

16. Yoshida, S.; Yamamoto, S.; Takamatsu, T. Rapid Commun. Mass Spectrom. 1998, 12, 535-544.

17. van Rooij, G. J.; Duursma, M. C.; Heeren, R. M. A.; Boon, J. J.; de Koster, C. G. J. Am. Soc. Mass Spectrom. 1996, 7, 449-457.

18. de Koster, C. G.; Duursma, M. C.; van Rooij, G. J.; Heeren, R. M. A.; Boon, J. J. Rapid Commun. Mass Spectrom. 1995, 9, 957-962.

19. Gooden, J. K.; Gross, M. L.; Mueller, A.; Stefanescu, A. D.; Wooley, K. L. J. Am. Chem. Soc. 1998, 120, 10180-10186.
20. Belu, A. M.; DeSimone, J. M.; Linton, R. W.; Lange, G. W.; Friedman, R. M. J. Am. Soc. Mass Spectrom. 1996, 7, 11-24.

21. Jackson, C. A.; Simonsick, W. J., Jr. Curr. Opin. Solid State Mater. Sci. 1997, 2, 661-667.

22. Montaudo, G. TRIP 1996, 4, 81-86.

23. Wu, K. J.; Odom, R. W. Anal. Chem. 1998, 70, 456A-461A.

24. Saf, R.; Mirtl, C.; Hummel, K. Acta Polym. 1997, 48, 513-526.

25. Parees, D. M.; Hanton, S. D.; Cornelio Clark, P. A.; Wilcox, D. A. J. Am. Soc. Mass Spectrom. 1997, 9, 282-291.

26. Hunt, S. M.; Sheil, M. M.; Derrick, P. J. Eur. Mass Spectrom. 1998, 4, 475-486.

27. Latourte, L.; Blais, J. C.; Tabet, J. C. Anal. Chem. 1997, 69 , 2742-2750.

28. Guittard, J.; Tessier, M.; Blais, J. C.; Bolbach, G.; Rozes, L.; Marechal, E.; Tabet, J. C. J. Mass Spectrom. 1996, 31, 1409-1421.

29. Yan, W.; Ammon, D. M., Jr.; Gardella, J. A., Jr.; Maziarz, E. P., III; Hawkridge, A. M.; Grobe, G. L., III; Wood, T. D. Eur. Mass Spectrom. 1998, 4, 467-474.

30. Schriemer, D. C.; Li, L. Anal. Chem. 1997, 69, 4169-4175.

31. Schriemer, D. C.; Li, L. Anal. Chem. 1997, 69, 4176-4183.

32. Axelsson, J.; Scrivener, E.; Haddleton, D. M.; Derrick, P. J. Macromolecules 1996, 29, 8875-8882.

33. Martin, K.; Spickermann, J.; Rader, H. J.; Mullen, K. Rapid Commun. Mass Spectrom. 1996, 10, 1472-1474.

34. Vitalini, D.; Mineo, P.; Scamporrino, E. Rapid Commun. Mass Spectrom. 1999, 13, 2511-2517.

35. Marie, A.; Fournier, F.; Tabet, J. C. Anal. Chem. 2000, 72, 5106-5114.

36. Schriemer, D. C.; Whittal, R. M.; Li, L. Macromolecules 1997, 30, 1955-1963.

37. Knochenmuss, R.; Lehmann, E.; Zenobi, R. Eur. Mass Spectrom. 1998, 4, 421-427.

38. Yalcin, T.; Schriemer, D. C.; Li, L. J. Am. Soc. Mass Spectrom. 1997, 8, 1220-1229.

39. Kanjolia, R. K.; Gupta, V. D. Z. Naturforsch 1980, 35b, 767-770.

40. Liu, X. M., Maziarz, E.P., III, Unpublished data.

41. Montaudo, M. S.; Puglisi, C.; Samperi, F.; Montaudo, G. Rapid Commun. Mass Spectrom. 1998, 12, 519-528.

42. Aaserud, D. J.; Prokai, L.; Simonsick, W. J., Jr. Anal. Chem. 1999, 71, 4793-4799.

43. Hanton, S. D.; Liu, M. X. Anal. Chem. 2000, 72, 4550-4554.

44. Xianwen, L.; Van Dongen, J. L. J. J. Mass Spectrom. 2000, 35, 1308-1312.

45. Nielen, M. W. F.; Buijtenhuijs, F. A. Anal. Chem. 1999, 71, 1809-1814.

46. Yun, H.; Olesik, S. V.; Marti, E. H. J. Micro. Sep. 1999, 11, 53-61.

47. Nielen, M. W. F. Anal. Chem. 1998, 70, 1563-1568.

48. Kassis, C. M.; DeSimone, J. M.; Linton, R. W.; Lange, G. W.; Friedman, R. M. Rapid Commun. Mass Spectrom. 1997, 11, 1462-1466.

49. Wallace, W. E.; Guttman, C. M.; Antonucci, J. M. Polymer 2000, 41, 2219-2226.

50. Wallace, W. E.; Guttman, C. M.; Antonucci, J. M. J. Am. Soc. Mass Spectrom. 1999, 10, 224-230.

51. Montaudo, G.; Montaudo, M. S.; Puglisi, C.; Samperi, F. Rapid Commun. Mass Spectrom. 1995, 9, 453-460. 